\title{
Assessment of job satisfaction among dentists working in different settings in the Tshwane metro
}

SADJ June 2019, Vol. 74 No. 5 p230 - p235

SA Matjila', TK Madiba², L Annandale-Dippenaar³ ${ }^{3}$ BG Olutola ${ }^{4}$

\section{SUMMARY}

\section{Introduction}

Job satisfaction of dentists is important, as dentists are critical in the delivery of comprehensive health care.

Whilst job satisfaction among dentists has been widely investigated elsewhere, it is poorly researched in South Africa.

Aims and objectives

To assess job satisfaction among dentists working in Tshwane district.

\section{Design}

A cross-sectional study.

\section{Methods}

Data were collected using a self-administered, validated, modified questionnaire adapted from the Dentists' Satisfaction Survey. Demographic data and the responses associated with job satisfaction in various settings were collected.

Author affiliations:

1. Sello A Matjila: B. Dent Ther, BDS, Dip Odont, MBL, PGD (Health Economics), Sefako Makgatho Health Sciences University, Pretoria, South Africa.

ORCID Number: 0000-0001-5446-6904

2. Thomas K Madiba: B. Dent Ther, BDS, DHSM, MChD (Community Dentistry), Department of Community Dentistry, School of Dentistry, University of Pretoria, Pretoria, South Africa. ORCID Number: 0000-0002-0171-0595

3. Lea Annandale-Dippenaar: DOAL (Applied Leadership), UGSM Monarch Business School, Zug, Switzerland, University of South Africa and Associate of the Ethics Institute. ORCID Number: 0000-0002-1801-0050

4. Bukola G Olutola: BDS, MSc (Epidemiology), School of Health Systems and Public Health, University of Pretoria, Pretoria, South Africa.

ORCID Number: 0000-0003-4114-8290

Corresponding author: Thomas Madiba

Department of Community Dentistry, School of Dentistry, University of Pretoria, Pretoria, South Africa.

P.O. Box 21497

Valhalla 0137

Tel: +27 (0)12 3192417 Email: thommy.madiba@gmail.com

Author contributions:

1. Sello A Matjila: Principal investigator, protocol, literature review, discussion and proof reading $-35 \%$

2. Thomas K Madiba: Protocol, discussion, manuscript preparation, abstract and proof reading $-30 \%$.

3. Lea Annandale-Dippenaar: Protocol, methods, manuscript preparation and proof reading - 25\%

4. Bukola G Olutola: Data analysis and writing of results $-10 \%$
Two of the questions were qualitative and open-ended. Data were analysed using statistical software package (STATA) version 10. Ethical clearance was obtained and all data were anonymous.

\section{Results}

The response rate was $77 \%$ and $53 \%(41)$ of respondents were female. Perception of income, relationships with patients and personal time were significantly positively associated with job satisfaction.

Patient happiness with treatment, the autonomy and flexibility of private practice and imparting knowledge also contributed to job satisfaction.

Unrealistic expectations of patients, long working hours, restricted funding and lack of equipment contributed to job dissatisfaction.

\section{Conclusion}

Overall the dentists were satisfied, however, satisfaction and dissatisfaction were conditional and influenced by factors such as perception of income and unrealistic patient expectations.

Keywords

Job satisfaction, dentists, settings.

\section{INTRODUCTION}

Morale in the workplace, or job satisfaction, has been the subject of much research. This is mainly because, either as an individual or as an important inter-related factor, job satisfaction forms a significant part of the literature in Human Resource Management. ${ }^{1}$

Importantly, job satisfaction influences productivity and performance, as well as the quality of life. One author defined the concept of job satisfaction as the pleasurable emotional state from the appraisal of one's job. ${ }^{2}$

Employee job satisfaction is recognised as a multifaceted construct that includes the feelings of employees about a variety of both intrinsic and extrinsic job elements. ${ }^{3}$

It has been reported that intrinsic factors are factors such as the nature of the job itself and the opportunity for growth, which are internally mediated rewards. ${ }^{3}$ 
The externally mediated rewards are, for example, pay and benefits, company policies and support, supervision and relationships with supervisor and co-workers, promotion and working conditions. ${ }^{4}$

Job satisfaction is essential in predicting the stability of systems, worker motivation and retention. Dentistry has been identified as one of the most stressful health professions. ${ }^{5}$

Most studies about job satisfaction among dentists have been conducted in Australia, the United Kingdom and Lithuania. ${ }^{6-10}$ However, not much is known about the factors that influence job satisfaction of dentists in South Africa.

The study therefore was aimed at assessing job satisfaction among dentists working in different settings in Tshwane district.

\section{METHODOLOGY}

A cross sectional descriptive survey was conducted in 2012, based on an adapted Dentists Satisfaction Survey ${ }^{11}$ in order to determine the factors influencing job satisfaction among dentists working in the private and public sectors in the Tshwane Municipality, which is situated in the north of Gauteng Province. Two qualitative open-ended question were added to the questionnaire.

Tshwane is composed of urban, peri-urban and rural settlements. There are two dental schools in the Municipality, namely the School of Oral Health Sciences at Sefako Makgatho Health Sciences University, and the School of Dentistry at the University of Pretoria. The South African Military Health Services also operate several oral health clinics in the area.

The study opted for a convenience sampling, targeting respondents from each occupational sector. The sample was stratified to comprise 25 academics, 25 private practitioners, 25 South African Military Oral Health personnel and 25 dentists working in government clinics, comprising a sample of 100 participants.

A self-administered, modified and validated questionnaire was used to collect the necessary data. ${ }^{11}$ The questionnaires posed enquiries on demographic data and factors that measured dimensions such as overall job satisfaction, perception of income, personal time, professional time and relationship with staff, opportunities for advancement, relationship with patients and standards of delivery of care.

In addition there were two questions of a qualitative nature, namely: "In your opinion, what aspects of your job satisfy you the most?" and "What aspects of your job dissatisfy you the most?"

To ascertain the internal reliability of the measuring tool, the Cronbach's alpha was calculated for each item to measure how closely the statement was identified in the factor and for the overall job facet. Items with poor internal reliability were discarded before the full statistical analysis.
The proposal was submitted to the University of South Africa School of Business Leadership and received ethical clearance. No personal details of the participants were recorded and all information was treated as strictly confidential.

\section{Statistical analyses}

The statistical analyses were performed using STATA (StataCorp, 2013. Stata Statistical Software: Release 13. College Station, TX, USA: StataCorp LP). Data were reduced using factor analysis into overall job satisfaction, perception, personal time, professional time, support staff, relationship with patients and delivery of care.

Bivariate analyses such as the T-test and correlations were carried out between overall job satisfaction and binary and continuous variables respectively.

Multivariate linear regression was then carried out using a backward deletion approach by starting with a full model of all independent variables regardless of whether they were significant predictors of overall job satisfaction (forced entry method).

Statistical significance was set at $p<0.05$ for multivariate linear regression. The responses from the two qualitative questions were categorised in terms of common themes.

\begin{tabular}{|c|c|c|}
\hline Characteristics & \multicolumn{2}{|c|}{$\%(n)$} \\
\hline \multicolumn{3}{|l|}{ Age (Years) } \\
\hline $20-29$ & 14.3 & (11) \\
\hline $30-39$ & 36.4 & (28) \\
\hline $40-49$ & 32.5 & (25) \\
\hline 50 and above & 16.9 & (13) \\
\hline \multicolumn{3}{|l|}{ Gender } \\
\hline Male & 46.8 & (36) \\
\hline Female & 53.3 & (41) \\
\hline \multicolumn{3}{|l|}{ Marital status } \\
\hline Single & 24.7 & (19) \\
\hline Married & 74.0 & (57) \\
\hline Divorced & 1.3 & (1) \\
\hline \multicolumn{3}{|l|}{ Highest level of education } \\
\hline BChD, BDS & 58.4 & (45) \\
\hline Postgraduate diploma & 18.2 & (14) \\
\hline Masters & 11.7 & (9) \\
\hline Specialization (MChD, MDent) & 9.1 & (7) \\
\hline Other & 2.6 & (2) \\
\hline \multicolumn{3}{|l|}{ Location } \\
\hline Urban & 81.3 & (52) \\
\hline Peri-urban & 18.8 & (12) \\
\hline \multicolumn{3}{|l|}{ Years of practice } \\
\hline Other & 51.4 & (38) \\
\hline Wellness site (15-19 yrs.) & 48.7 & (36) \\
\hline
\end{tabular}

\section{RESULTS}

Of the sample of $100,77(77 \%)$ participated and of these $53 \%(41)$ were female. The ages ranged from 20 to 69 years, with $69 \%$ in the range between $30-49$ years, while $74 \%$ of the respondents were married (Table 1 ). 
As to the place of work, $44 \%, 32 \%, 17 \%$ and $7 \%$ were in private, public, academia and the military health services respectively. Among those who reported that they were working in more than one sector, 55.6\% indicated that the public sector was their main practice area, leaving $44.4 \%$ who responded that their primary employment was in private practice. The respondents' years of practice ranged to greater than 21 years.

Using factor analysis, seven groupings were discovered from the data. These included;

- Overall job satisfaction (Cronbach's alpha=0.87\%),

- Perception of income (Cronbach's alpha=0.87\%),

- Personal time (Cronbach's alpha=0.97\%),

- Professional time (Cronbach's alpha=0.74\%),

- Support staff (Cronbach's alpha=0.83\%),

- Patient relations (Cronbach's alpha=0.72\%),

- and Delivery of care ( Cronbach's alpha=0.76\%).

\begin{tabular}{lcc}
$\begin{array}{l}\text { Table 2. Correlation between job satisfaction and other characteristics } \\
\text { Characteristics }\end{array}$ & $\begin{array}{c}\text { Pearson } \\
\text { correlation coefficient }\end{array}$ & $p$-value \\
\hline Perception of income & 0.41 & $<0.001$ \\
\hline Personal time & 0.24 & 0.040 \\
\hline Professional time & 0.14 & 0.260 \\
Support staff & 0.18 & 0.130 \\
Patient relations & 0.31 & 0.010 \\
Delivery of care & 0.22 & 0.070
\end{tabular}

Table 3. Bivariate relationship of socio-demographic factors by job satisfaction

\begin{tabular}{|c|c|c|c|}
\hline $\begin{array}{l}\text { Characteristics } \\
\text { Age }\end{array}$ & \multicolumn{2}{|c|}{ Mean (SE) } & $\begin{array}{c}\boldsymbol{p} \text { - value } \\
0.44\end{array}$ \\
\hline$<50$ years & 24.9 & $(0.72)$ & \\
\hline$\geq 50$ years & 26.0 & (1.26) & \\
\hline Gender & & & 0.67 \\
\hline Male & 25.3 & (0.93) & \\
\hline Female & 24.8 & $(0.86)$ & \\
\hline Location & & & 0.01 \\
\hline Urban & 24.2 & $(0.80)$ & \\
\hline Peri-urban & 28.1 & (1.08) & \\
\hline Years of practice & & & 0.21 \\
\hline$\leq 10$ years & 25.7 & (0.92) & \\
\hline$>10$ years & 24.1 & $(0.88)$ & \\
\hline Main practice & & & 0.64 \\
\hline Private & 25.2 & (1.34) & \\
\hline Public & 26.1 & (1.23) & \\
\hline
\end{tabular}

Table 4. Multiple linear regression predicting factors associated with job satisfaction

Characteristics Odds ratio $95 \%$ Confidence interval

Age

\begin{tabular}{lll}
\hline$<\mathbf{5 0}$ years & 1.0 & \\
$\mathbf{2} \mathbf{5 0}$ years & 1.21 & $1.05-1.40$ \\
\hline $\begin{array}{l}\text { Years of practice } \\
\mathbf{1 0} \text { years }\end{array}$ & 1.0 & \\
$>\mathbf{1 0}$ years & 0.85 & $0.77-0.95$ \\
\hline Perception of income & 1.02 & $1.01-1.03$ \\
\hline Years of practice & 1.03 & $1.01-1.05$ \\
\hline
\end{tabular}

There was a correlation between overall job satisfaction and perception of income. The higher the respondents perceived their income, the higher the job satisfaction (Table 2).

Participants with practices in the peri-urban areas reported better job satisfaction than did those in the urban area. However, gender and number of years of practice did not have any association with job satisfaction (Table 3).

Multiple linear regression predicting factors associated with linear job satisfaction revealed that dentists who were 50 years and above were more likely to be more satisfied with their jobs when compared with those who were below $50(\mathrm{OR}=1.21$; $95 \%$ confidence interval $=1.05-1.40$ ). Also, those dentists who reported more positively on the perception of income $(\mathrm{OR}=1.02$; $95 \%$ confidence interval $=1.01-1.103)$, had a higher job satisfaction (Table 4).

It was also found that those dentists who reported to be happy with their relationship with patients $(O R=1.03$; 95\% Confidence interval $=1.01-1.05)$, were more satisfied with their job (Table 4).

\section{Qualitative analysis}

Questions 8 and 9 of the questionnaire were open-ended. For thorough analysis, underlying themes were identified from the various responses. The results are reported below:

\section{Question 8: In your opinion, what aspects of your job satisfy you the most?}

Major themes identified were as follows:

\section{Patients' satisfaction}

A number of respondents reported that seeing patients satisfied with the treatment they had received made the practitioner more satisfied about his/her job. The tone of the responses was:

A happy and satisfied patient when leaving my surgery - Male, Private Practice

Reaching out to a person who came with a dental problem and who is able to leave with a smile and satisfaction - Female, Public Service

Seeing a happy and satisfied patient is most fulfilling - Male, Academic

\section{Interaction}

Interaction with patients, colleagues and students are the sub-themes that stood out amongst the responses from the dentists surveyed. The respondents reported that interacting with the groups mentioned above enhanced their job satisfaction.

Interaction with students - Female, Academic

Interacting with fellow colleagues - Female, Academic

Interacting with patients - Male, Military Health Services 


\section{Helping patients}

The respondents reported that they were satisfied when they could see that they have helped a patient.

It feels good to know that you have removed someone's pain and improved someone's aesthetics

- Male, Private Practice

Solving patients' problem - Female, Academic

To provide help to my patients, mostly to alleviate their pain - Male, Public Sector

\section{Teaching and research}

Respondents from the academic sector reported that their job satisfaction was enhanced by imparting knowledge to their students and by research.

I also enjoy post-graduate teaching and training

- Male, Academic

Working with students and doing research

- Female, Academic

Career development in research - Female, Academic

Helping postgraduate students with big complex cases - Female, Academic

\section{Educating patients and oral health promotion}

The opportunity of being able to educate patients was reported to be a factor that also enhanced job satisfaction.

Being able to promote oral health to the community especially the elderly and the schoolchildren

- Female, Public Sector

The amount of dental education and knowledge I provide to my patients to improve their dental IQ

- Female, Private Practice

Improving oral healthcare outcomes for patients through educating patients and seeing results

- Female, Academic

\section{Autonomy}

Respondents from private practice reported that the autonomy associated with the running of their practices enhanced their job satisfaction.

Clinical freedom - the ability to decide on treatment planning issues - Female, Private Practice

In my work I am my own boss - Male, Private Practice

Working for myself, determining the working hours

- Male, Private Practice

Flexibility of time - I am the boss - Male, private practice

\section{Relationships with colleagues}

A number of respondents reported that having a good relationship with colleagues was pivotal in enhancing their job satisfaction.
Having a good working relationship with my colleagues and being part of a team - Female, Academic

Good support from staff makes my work easier to perform despite the long working hours - Male, Private Practice

Friendly, helpful co-workers - Female, Academic

\section{Treatment outcomes}

Dentistry is a very clinically oriented profession. A number of respondents reported that having favourable treatment outcomes gave them satisfaction in their job. One respondent's response was:

With orthodontics, the end results are life changing for a patient e.g. I have treated a 9yr old class II Div 1 patient who was being teased at school and after the treatment, the mother came back to tell me that her son is now confident and his school work has improved and he was even chosen as a class prefect - Male, Academic

\section{Question 9: What aspects of your job dissatisfy you the most?}

Major themes identified were as follows:

\section{Patients' expectations}

A number of respondents reported that unrealistic expectations of patients contributed to their dissatisfaction about their job.

Occasional unrealistic expectations from patients

- Female, Military Health Services

Ungrateful patients

- Female, Private Practice

\section{Long working hours}

A number of respondents reported that long working hours contributed to their dissatisfaction about their job.

Having to work hard to fulfil your dreams. Not having enough time to take a holiday - Female, Private Practice

Long working hours, non-flexible time - Female, Academic

\section{Lack of equipment and materials}

A number of respondents from both the public sector and academia reported that lack of equipment and dental materials necessary for them to carry out their duties contributed to their dissatisfaction with their job.

The technical aspects - not enough equipment, lack of maintenance and repairs of equipment, outdated equipment - Male, Public Sector

Lack of resources e.g. gloves. Outdated equipment - Female, Public Sector

Lack of resources for teaching, training, and research and service delivery makes working in this field a very frustrating exercise - Male, Academic

\section{Lack of career development}

A number of the respondents reported that their careers were stagnant and that contributed to their dissatisfaction about their jobs. 
Not receiving further training in other fields such as endodontics, surgical removal of teeth, which are not provided in public sector/most primary health care centres - Female, Public Sector

Lack of opportunities to advance or enrich our skills - Female, Public Sector

\section{Heavy workload}

A number of respondents reported that the heavy workload contributed to their job dissatisfaction.

Having to deal with a large number of patients to have their teeth extracted on a daily basis

- Female, Public Sector

Stress caused by too heavy workload (too many patients) - Male, Public Sector

\section{Lack of support from management}

Targets set by superiors that are impossible to meet consistently - Female, Military Health Services

Lack of support from top management to get the best quality instruments and equipment in order to make a proper diagnosis and treatment - Female, Public Sector

The public sector is negligent in promoting people on time, and operates under the premise that its employees can be shoved aside for trivial matters

- Male, Public Sector

\section{Dealing with third party funders/medical aid schemes}

A number of respondents from private practice reported that dealing with medical aid schemes contributes to dissatisfaction about the job.

Restriction in funding allocated for dentistry by Medical Aids often means that I have to provide compromised treatment and not necessarily the best treatment for that patient - Male, Private Practice

Most medical aid benefits covering the cost of both basic and special dentistry treatments are too little. Authorisation processes required by most medical aids vary - Male, Private Practice

Medical aid regulations or exclusions that don't cover for things that are clinically warranted. Obviously the fees that I charge are at times too low, but I am guided by my market - Male, Private Practice

\section{Inability of patients to pay for dental treatment}

A number of respondents reported that the inability of patients to pay for dental treatment contributed to their job satisfaction about their job.

Always finances stand in the way of providing good quality work for patients visiting our training centre

- Male, Academic

Patient not able to afford the cost of treatment

- Female, Private Practice

\section{DISCUSSION}

The age distribution of the respondents in the study pointed towards the national trend. The Quarterly Labour Force Survey of the second quarter of 2012 indicated that amongst employed South Africans, the majority are between the ages of 30 and 50 years. ${ }^{12}$

The results showed that there were no demographic characteristics which were associated with job satisfaction. Similar studies in the USA ${ }^{13}$ and the $\mathrm{UK}^{8}$ in which the same survey instrument was used, found that there were few or no demographic and practice characteristics which related to job satisfaction. However, a USA study identified lower job satisfaction among rural dentists than those who practiced in urban areas. ${ }^{13}$ In contrast; this study identified higher job satisfaction among dentists who practiced in peri-urban areas.

Perception of income, relationship with patients and personal time were found to be significantly associated with job satisfaction. These findings are similar to the study done on factors related to job satisfaction among South Korean dentists. ${ }^{10}$

The relationship between job satisfaction and professional time was also found to be insignificant. These findings were in contrast to the study among Canadian orthodontists. ${ }^{14}$ Responses from the survey demonstrate that dentists working in different settings are as a group generally satisfied with intrinsic factors such as helping patients and seeing patients satisfied with their treatments.

Dentists working in the academic setting reported that their job satisfaction was enhanced by imparting knowledge to their students and doing research whilst those in private practice reported that their satisfaction was enhanced by the autonomy and the flexibility associated with being self-employed.

A number of respondents reported that having favourable treatment outcomes resulted in job satisfaction. These findings are consistent with findings from a study in Holland which reported that immediate results, aesthetics and long-term results of working with patients enhanced job satisfaction of dentists. ${ }^{15}$

A number of respondents from different settings reported that the unrealistic expectations of patients contributed to dissatisfaction. These results are similar to a study that reported that higher patient expectations and higher targets for the provision of dental care puts increased demands on dentists. ${ }^{16}$

A number of respondents reported that they have to put in long hours, which contributed to job dissatisfaction. Respondents from private practice complained that they have to work hard to fulfil their dreams whilst those from the academic field reported that their working hours are long and lack flexibility. One study reported that doctors and dentists who take on a teaching role in addition to their clinical role may increase their levels of stress, but there is also evidence that this dual role may reduce job-related stress. ${ }^{17}$ 
The frustration with the lack of dental equipment and materials is consistent with a study that found that lack of equipment, malfunctioning and delay in repairs of equipment remains a considerable source of frustration. ${ }^{18}$

Dentistry in South Africa has limited opportunites in the specialities and the majority of dentists are finding it difficult to advance their careers. A number of respondents reported being frustrated about their lack of career development.

Private practitioners, in particular, were dissatisfied about third party funders restricting dental benefits to the patient, thereby denying their autonomy in making treatment decisions for their patients. Dentists reported that they have to deal with patients who cannot afford their services and that hinders them in the provision of quality care to their patients.

\section{CONCLUSIONS}

On overall, the dentists in Tshwane are more satisfied than dissatisfied with their jobs. The satisfaction is however; conditional.

Perception of income, relationship with patients and personal time were significantly associated with job satisfaction. Intrinsic factors such as helping patients and seeing patients satisfied with their treatments contributed to satisfaction.

For dentists working in the academic setting their satisfaction was positively correlated with imparting knowledge to their students and in pursuing research, whilst those in private practice valued the autonomy and the flexibility associated with being self-employed.

Job dissatisfaction was positively correlated with unrealistic patients' expectations, long working hours, lack of career progression, restricted funding from funders and lack of equipment.

\section{Limitations of the study}

This study finding should be interpreted with caution, as there are some design limitations. For example, this was a cross-sectional study, therefore causality cannot be inferred.

The research was conducted on a small sample size and therefore, the results cannot be generalised either with regard to the Tshwane Metropolitan as a whole or to other areas. The study should have involved more participants both at different levels and in Tshwane Metropolitan as well as other locations.

Despite limitations, the current study provided useful information that may inform the design of further studies.

\section{Recommendations}

It is recommended that the study be extended to a national project on a large sample regarding job satisfaction for dentists, providing a more in-depth understanding of how dentists in South Africa feel about their jobs.
Management in the public sector needs to review the working conditions of dentists and the procurement of dental equipment so that dental practitioners can practice their profession without limitations.

\section{Financial support and sponsorship}

None.

\section{Conflicts of interest}

There are no conflicts of interest.

\section{References}

1. Trivellas P, Dargenidou D. Organisational culture, job satisfaction and higher education service quality: The case of the Technological Educational Institute of Larissa. The TQM Journal. 2009; 21(4):382-99.

2. Locke EA. What is job satisfaction? Organizational Behavior and Human Performance. 1969; 4(4):309-36.

3. Misener TR, Haddock KS, Gleaton JU, Ajamieh ARA. Toward an international measure of job satisfaction. Nurs Res. 1996; 45(2): 87-91.

4. McKenna EF. Business Psychology and Organizationa Behaviour. First published 2012 by Psychology Press 27 Church Road, Hove, East Sussex, BN3 2FA Simultaneously published in the USA and Canada by Routledge (or) Psychology Press. 2012.

5. Myers H, Myers L. 'It's difficult being a dentist': stress and health in the general dental practitioner. Br Dent J. 2004; 197(2): 89 .

6. Puriene A, Aleksejuniene J, Petrauskiene J, Balciuniene I, Janulyte V. Self-perceived mental health and job satis faction among Lithuanian dentists. Ind Health. 2008; 46(3): 247-52.

7. Luzzi L, Spencer A. Job satisfaction of the oral health labour force in Australia. Aust Dent J. 2011; 56(1):23-32.

8. Gilmour J, Stewardson D, Shugars D, Burke F. An assessment of career satisfaction among a group of general dental practitioners in Staffordshire. Br Dent J. 2005; 198 (11):701.

9. Harris RV, Ashcroft A, Burnside G, Dancer JM, Smith D, Grieveson B. Facets of job satisfaction of dental practitioners working in different organisational settings in England. Br Dent J. 2008; 204(1): E1.

10. Jeong SH, Chung JK, Choi YH, Sohn W, Song KB. Factors related to job satisfaction among South Korean dentists. Community Dent Oral Epidemiol. 2006; 34(6):460 - 6.

11. Shugars DA, Hays RD, DiMatteo MR, Cretin S. Development of an instrument to measure job satisfaction among dentists. Med Care. 1991:728-44.

12. Statistics SA. Pretoria: Statistics South Africa. 2012. Quarterly labour survey $2^{\text {nd }}$ quarter.

13. Wells A, Winter P. Influence of practice and personal characteristics on dental job satisfaction. J Dent Educ. 1999; 63(11):805-12.

14. Roth SF, Heo G, Varnhagen C, Glover KE, Major PW. Job satisfaction among Canadian orthodontists. Am J Orthod Dentofacial Orthop. 2003; 123(6):695-700.

15. Gorter R, Te Brake J, Eijkman M, Hoogstraten J. Job resources in Dutch dental practice. Int Dent J. 2006; 56(1):22-8.

16. Kaney S. Sources of stress for orthodontic practitioners. $\mathrm{Br} J$ Orthod. 1999.

17. Rutter H, Herzberg J, Paice E. Stress in doctors and dentists who teach. Med Educ. 2002; 36(6):543-9.

18. Naidoo S. Community Dental Service-four years on: Communication. South African Dental Journal. 2007; 62(6): $250-3$ 